\title{
Parámetros físicos del suelo en condiciones no perturbadas y bajo laboreo(1)
}

\author{
Laura Alejandra Ferreras ${ }^{(2)}$, Juan Jose De Battista ${ }^{(3)}$, Alfredo Ausilio(2) y Carlos Pecorari(4)
}

\begin{abstract}
Resumen - Se estudió el efecto de dos sistemas de labranza sobre algunas propiedades del suelo y el rendimiento de soja. La experiencia se realizó sobre un Argiudol típico durante 1996. Los tratamientos fueron: siembra directa (SD), labranza vertical (LV) y un control no laboreado (T). Se determinó: densidad del suelo (DS); densidad máxima (DSMAX); compactación relativa (CR); porosidad estructural (PE); carbono orgánico total (COT), humificado $(\mathrm{COH})$ y libre $(\mathrm{COL})$ y humedad gravimétrica (HG). Se realizó el perfil cultural en LV y SD. La DS en SD fue significativamente mayor (P $\leq 0,01)$ en superficie y en profundidad (1,22 y $1,37 \mathrm{Mg} \mathrm{m}^{-3}$, respectivamente). La PE en superficie fue $\mathrm{LV}>\mathrm{T}>\mathrm{SD}$, mientras que en profundidad fue $\mathrm{T}>\mathrm{LV}>\mathrm{SD}$. Hubo relación inversa entre $\mathrm{CR}$ y $\mathrm{PE}\left(\mathrm{r}^{2}=0,87\right)$. El perfil cultural mostró un $40 \%$ de agregados sin porosidad interna en SD. En superficie T presentó mayor COT, COH y COL. La DSMAX se correlacionó negativamente con el COT $\left(\mathrm{r}^{2}=0,88\right)$. Al inicio del cultivo, SD presentó mayor HG $(\mathrm{P} \leq 0,01)$ que $\mathrm{LV}$; en floración $\mathrm{LV}$ presentó mayor $\mathrm{HG}(\mathrm{P} \leq 0,01)$. Si bien SD presentó mayor COT, $\mathrm{COH}$ y COL que LV, la mayor compactación observada en SD pudo incidir negativamente en el rendimiento del cultivo (fue el 50\% de LV).
\end{abstract}

Términos para índice: labranza cero, labranza vertical, densidad del suelo, porosidad, compuestos orgánicos, compactación del suelo.

\section{Physical parameters of a soil under minimum tillage and non-disturbed conditions}

Abstract - The effect of two tillage systems on soil properties and soybean yield was studied. The experiment was carried out on a Typic Argiudoll during 1996. Treatments were: no-tillage (NT), chisel plow (CP) and a control not sown (CNS). Soil bulk density (SBD), maximum bulk density (MAXDB), relative compaction (RC), structural porosity (SP), total organic carbon (TOC), humified organic carbon (HOC), free organic carbon (FOC) and water content were evaluated. Soil profile analysis was performed for both tillage systems. Results of SBD in NT showed significant differences both at surface and in depth (1.22 and $1.37 \mathrm{Mg} \mathrm{m}^{-3}$, respectively). The SP at both sampling depths was in the order $\mathrm{CP}>\mathrm{CNS}>\mathrm{NT}$ and $\mathrm{CNS}>\mathrm{CP}>\mathrm{NT}$, respectively. $\mathrm{RC}$ was inversely related to the $\mathrm{SP}\left(\mathrm{r}^{2}=0.87\right)$. Soil profiles showed a $40 \%$ of aggregates without internal porosity in NT. TOC, HOC and FOC on surface was higher $(\mathrm{P} \leq 0,01)$ in $\mathrm{T}$ as respect $\mathrm{NT}$ and $\mathrm{CP}$. MAXBD was negatively correlated to TOC $\left(r^{2}=0.88\right)$. Water content in NT was higher $(\mathrm{P} \leq 0,01)$ than in $\mathrm{CP}$ at the initial stages, while it was higher $(\mathrm{P} \leq 0.01)$ in $\mathrm{CP}$ at flowering. Though TOC, HOC and FOC were slightly higher in NT than CP, higher compaction in NT could negatively influence on crop yield (50\% lower than $\mathrm{CP}$ ).

Index terms: zero tillage, ploughing, soil density, porosity, organic compounds, soil compaction.

\footnotetext{
(1) Aceptado para publicación en 13 de enero de 2000.

(2)Universidad Nacional de Rosario (UNR), Facultad de Ciencias Agrarias, C.C. 14, Zavalla 2123, Santa Fe, Argentina.

E-mail: lferrera@sede.unr.edu.ar, aausilio@sede.unr.edu.ar

${ }^{(3)}$ Instituto Nacional de Tecnología Agropecuaria (INTA), Estación Experimental Agropecuaria (EEA), C.C. 6, Concepción del Uruguay 3260, Entre Ríos, Argentina. E-mail: normajuan@teletel.com.ar

(4) INTA-EEA, C.C. 22, Rafaela 2300, Santa Fe, Argentina. E-mail:cpecora@inta.gov.ar
}

\section{Introducción}

La intensificación de los sistemas de cultivo en la Argentina se aceleró en el transcurso de las dos últimas décadas. En la Región Pampeana esto trajo como consecuencia una reducción de la superficie cubierta con praderas semipermanentes y un crecimiento de las áreas dedicadas a las secuencias agrícolas anuales. Estos sistemas basados en una agricultura continuada e intensiva corren un severo riesgo de pérdida 
de la producción como consecuencia del deterioro del recurso suelo (Pizarro et al., 1992). Esto puede producir una disminución de los rendimientos de los cultivos, aunque en algunos casos, no se visualiza totalmente debido al uso de agroquímicos y a las mejoras genéticas y tecnológicas (Pecorari, 1988).

Una alternativa para disminuir el proceso de degradación del suelo, es implementar sistemas de rotaciones agrícolo-ganaderos adecuados para cada situación, aunque actualmente estos sistemas no se consideran rentables. Por lo tanto, sería necesario explorar alternativas que permitan incrementar significativamente el período agrícola y a la vez preservar el recurso suelo (Coscia, 1989).

Algunos autores, en forma general, proponen la utilización de labranzas conservacionistas como una forma de reducir o evitar los efectos negativos de la agricultura continuada, tratando de restituir las buenas condiciones estructurales del suelo (Larney \& Kladivko, 1989). Según los datos presentados por Marelli (1994), la superficie agrícola bajo siembra directa en la Argentina cubría 150.000 ha en 1990, 500.000 ha en 1992 y en 1993 creció a 900.000 ha sobre una superficie total de 12 millones de hectáreas sembradas con los cultivos de soja, maíz y trigo.

Si bien la superficie sembrada bajo siembra directa se ha ido incrementando, en algunos establecimientos agrícolas de la Región Pampeana Norte se han presentado reducciones de los rendimientos en los cultivos realizados bajo esta práctica, factor que podría influir en su abandono.

Existe información con resultados dispares con respecto al efecto de los distintos sistemas de labranza sobre las propiedades físicas y químicas del suelo. Si bien, las condiciones de suelo, la rotación de cultivos y el tiempo desde que se aplican las labores varían, numerosos autores concluyen que bajo siembra directa, se produce un incremento en la compactación, resistencia a la penetración y contenido de agua para la capa arable (Nesmith et al., 1987; Mahboubi et al., 1993; Chagas et al., 1994). El incremento en la compactación podría restringir el abastecimiento de agua, aire, y la disponibilidad de nutrimentos (Oussible et al., 1992).

En numerosos trabajos de investigación se menciona, para un amplio rango de tipos de suelo, un menor crecimiento vegetativo de los cultivos bajo siembra directa, en comparación con cultivos sembrados en suelos con algún laboreo (Cornish \& Lymbery, 1987; Schmidt \& Belford, 1994). Estos autores atribuyeron como causa principal de la reducción del crecimiento del cultivo a factores de suelo de índole físico y biológicos.

Por otro lado, como una consecuencia favorable de estos sistemas que dejan el rastrojo en superficie se encontró que hay un incremento en la proporción de carbono orgánico y en la estabilidad de los agregados (Mahboubi et al., 1993; Chagas et al., 1994). Relacionando este aumento en la proporción de materia orgánica con la compactación, se hallaron en superficie valores de porosidad total mayores en suelos no trabajados, en comparación con los arados (Lal et al., 1994). Asimismo, Thomas et al. (1996) hallaron por debajo de esa capa rica en materia orgánica una zona más empobrecida donde aumentaba la compactación.

El objetivo de este trabajo fue evaluar el efecto de dos sistemas de labranza (siembra directa y labranza vertical) sobre las propiedades físicas de un Argiudol típico del Sur de Santa Fe con historia agrícola prolongada y su incidencia sobre el rendimiento del cultivo de soja.

\section{Material y Métodos}

La experiencia se llevó a cabo en un establecimiento agrícola ubicado en la localidad de Casilda, Departamento Caseros, provincia de Santa $\mathrm{Fe}$, Argentina $\left(33^{\circ} \mathrm{S}\right.$; $61^{\circ} \mathrm{W}$ ), durante el año 1996, sobre el cultivo de soja.

El suelo se clasifica como Argiudol típico serie Casilda, fina, illítica, térmica. El perfil de este suelo presenta un horizonte superficial de textura franco limosa (20\% arci1la, $70 \%$ limo, $10 \%$ arenas muy fina y fina). Se detallan a continuación algunas propiedades del horizonte superficial: espesor $=20 \mathrm{~cm}$, capacidad de intercambio catiónico $=16,7 \mathrm{cmol} \mathrm{kg}^{-1} ; \mathrm{pH}$ agua $(1: 2,5)=6,2$.

Se dispuso de dos situaciones: un suelo sin laboreo correspondiente al parque del establecimiento, el cual se consideró como tratamiento testigo (T). En la otra situación se realizó agricultura continua en forma convencional durante 30 años, pasando a labranza vertical en 1980. A partir del año 1991 se implementaron dos sistemas de labranza: siembra directa (SD) y labranza vertical (LV). Las rotaciones de cultivos para ambos tratamientos se detallan a continuación: 1991/92, trigo/soja; 1992/93, soja; 1993/94, maíz; 1994/95, trigo/soja; 1995/96, maíz; 1996/97, soja. 
La superficie destinada para cada uno de los tratamientos (siembra directa, labranza vertical y testigo) fue de aproximadamente 4 ha.

La secuencia de labores realizadas para el tratamiento LV fue la siguiente: rastra de discos a $10 \mathrm{~cm}$ de profundidad (junio); arado de cincel a $20 \mathrm{~cm}$ de profundidad junto con rastra de dientes (agosto); cultivador (octubre); rabasto y rastra de dientes (noviembre) y siembra.

Las malezas presentes en LV fueron controladas mediante la aplicación de trifluralina $\left(1,6 \mathrm{~L} \mathrm{ha}^{-1}\right)$ en presiembra, y propaquizafop $\left(0,6 \mathrm{~L} \mathrm{ha}^{-1}\right)$ en postemergencia. La incorporación del herbicida de presiembra se realizó a principios del mes de noviembre, utilizando rastra de dientes. Para el control de malezas en SD se aplicó glifosato $\left(3 \mathrm{~L} \mathrm{ha}^{-1}\right)$ en presiembra, y bentazon $\left(1 \mathrm{~L} \mathrm{ha}^{-1}\right)+$ propaquizafop $\left(0,6 \mathrm{~L} \mathrm{ha}^{-1}\right)$ en postemergencia.

La siembra se efectuó el 12 de noviembre, utilizando la variedad de soja N 5780 (24 granos/metro linear).

Se midió la densidad de volumen del suelo (DS) por el método del cilindro (Blake \& Hartge, 1986). Se extrajeron muestras de suelo sin disturbar al inicio del ciclo del cultivo (10/12/96), teniendo en cuenta las siguientes profundidades: de $0-6 \mathrm{~cm}$ y $10-16 \mathrm{~cm}$. Se extrajeron diez cilindros por profundidad y por cada tratamiento, utilizándose cilindros de $7 \mathrm{~cm}$ de diámetro y $6 \mathrm{~cm}$ de altura.

La densidad del suelo máxima (DSMAX) se determinó por medio del Test Proctor (Proctor, 1933), según las normas de la AASHO T-99 (American Association of State Highway Officials). Se utilizaron muestras de suelo considerando las siguientes profundidades: de $0-6 \mathrm{~cm}$ y de $6 \mathrm{~cm}$ al límite inferior del horizonte Ap $(20 \mathrm{~cm})$, para SD, LV y T. Las muestras se subdividieron en tres fracciones iguales y luego de humedecerlas (24 horas antes del análisis), se colocaron sucesivamente dentro del mortero $\left(0,947\right.$ dm ${ }^{3}$ de capacidad). La compactación se realizó capa por capa, mediante 25 golpes de maza $(2,5 \mathrm{~kg}$ de peso, lanzada a una altura de $30,5 \mathrm{~cm}$ del nivel de la muestra). Con los valores de densidad y de humedad se trazó la curva de compactación correspondiente a cada muestra. Se calculó la compactación relativa $(\mathrm{CR})$ dividiendo la DS actual por la DSMAX determinada por el test Proctor, expresando el resultado en porcentaje (Soane et al., 1981).

Para la determinación de la densidad textural (DTEX) se utilizó el método de Monnier et al. (1973). Se extrajeron muestras de suelo sin disturbar (0-6 y 6-20 cm de profundidad), al inicio del ciclo del cultivo. Las muestras fueron secadas al aire y se reservó la fracción de agregados comprendida entre 3 y $2 \mathrm{~mm}$ de diámetro. Con los valores obtenidos de DTEX se calcularon las porosidades estructural y textural de acuerdo a las siguientes fórmulas:

DTEX = P (DQ / (E- Eo))

$\mathrm{PE}=(1-\mathrm{DS} / \mathrm{DTEX}) \times 100$
PTEX $=$ PT - PE

donde

$\mathrm{P}=$ peso de la muestra seca,

$\mathrm{E}=$ empuje de la celda con los agregados,

$\mathrm{DQ}=$ densidad del querosene,

Eo = empuje de la celda vacía,

$\mathrm{PE}=$ porosidad estructural,

$\mathrm{DS}=$ densidad de volumen del suelo,

PTEX = porosidad textural,

$\mathrm{PT}=$ porosidad total.

Se efectuó el mapeo de los diferentes estados estructurales de acuerdo con la metodología del perfil cultural (Manichon, 1982, 1987) al momento de floración del cultivo. Se abrieron trincheras de $0,6 \mathrm{~m}$ de profundidad, $1 \mathrm{~m}$ de ancho y 1,3 m de longitud en los tratamientos SD y LV. Se realizó la delimitación y cartografiado de las unidades estructurales de acuerdo con el estado interno y la disposición de los terrones, expresando los resultados en porcentaje del área ocupada por cada estado estructural respecto del total. A continuación se detallan los niveles de organización determinados:

a) Primer nivel: Estado interno de los terrones: $\Gamma$ - caras rugosas con porosidad visible; $\Delta$ - caras lisas, de aspecto continuo, sin porosidad visible; $\theta$ - próximo a $\Delta$, pero con fisuras incipientes.

b) Segundo nivel: Disposición de los terrones: F - estado fragmentario con terrones individualizados; SF - estado fragmentario con terrones adherentes; $\mathrm{V}$ - cavidades; M - estado masivo; $\mathrm{Sd}$ - terrones difícilmente discernibles.

c) Tercer nivel: Reagrupamiento de las modalidades de disposición: óptimo $(\mathrm{O})$ - dominan las disposiciones $\mathrm{F}$ y SF, con abundante tierra fina; bloques (B) - dominan M y FV (terrones separados por cavidades grandes); compacto (C) - estado continuo, dominan M y Sd.

La determinación de carbono orgánico se realizó por el método de Walkley-Black (Black, 1965). Las fracciones orgánicas (libre y ligada o humificada) se separaron por el método de fraccionamiento por tamizado (Andriulo et al., 1990), determinando el carbono orgánico por el método de Walkley-Black. La metodología propuesta por Andriulo et al. (1990) consiste en separar granulométricamente la materia orgánica libre de la ligada, tamizando la muestra de suelo por un tamiz con una abertura de malla de $0,1 \mathrm{~mm}$. A partir de $0,1 \mathrm{~mm}$ no se hallan residuos vegetales entre microagregados, marcando una significativa separación entre restos orgánicos y complejos organo-minerales.

Se obtuvieron siete muestras compuestas al inicio del ciclo del cultivo (10/12/96), considerando las mismas profundidades de muestreo que se utilizaron para densidad textural. 
Se midió el contenido de agua (HG) en el horizonte superficial al inicio y durante el período crítico del cultivo (floración - fructificación) a través del método gravimétrico (Gardner, 1986). Se extrajeron diez muestras por tratamiento de labranza y por profundidad $(0-10$ y $10-20 \mathrm{~cm})$.

El rendimiento en grano fue medido mediante la cosecha mecánica de cada una de las unidades experimentales (SD y LV).

Los datos fueron analizados utilizando Statistical Analysis System (SAS Institute, 1985). El análisis de variancia empleado fue a dos factores (tratamiento y profundidad) con igual número de muestras (Neter et al., 1985), utilizando el procedimiento General Linear Model (GLM). La separación de medias entre tratamientos fue obtenida por el test de Duncan (Steel \& Torrie, 1986).

\section{Resultados y Discusión}

Los resultados de DS mostraron diferencias estadísticamente significativas $(\mathrm{P} \leq 0,01)$ entre tratamientos y entre profundidades (Cuadro 1), coincidiendo con lo reportado por Nesmith et al. (1987) y Mahboubi et al. (1993). El análisis de la interacción tratamiento $\mathrm{x}$ profundidad, fue también estadísticamente significativo $(\mathrm{P} \leq 0,01)$.

Los resultados de DSMAX medida por medio del test Proctor se presentan en el Cuadro 2. Se observa una diferencia entre las muestras provenientes del lote agrícola (SD y LV) en comparación con el testigo (T).

Con los resultados de DS actual y DSMAX se calculó la CR. Los valores de CR en superficie y profundidad correspondientes a SD resultaron los más elevados, mientras que LV en superficie y $\mathrm{T}$ en am-

Cuadro 1. Valores promedios de densidad de volumen del suelo $\left(\mathrm{Mg} \mathrm{m}^{-3}\right)$ en superficie y profundidad, en los tratamientos siembra directa, labranza vertical y testigo ${ }^{(1)}$.

\begin{tabular}{lcc}
\hline Tratamiento & \multicolumn{2}{c}{ Profundidad $(\mathrm{cm})$} \\
\cline { 2 - 3 } & $0-6$ & $10-16$ \\
\hline Siembra directa & $1,22 \mathrm{a}$ & $1,37 \mathrm{a}$ \\
Labranza vertical & $1,04 \mathrm{~b}$ & $1,32 \mathrm{a}$ \\
Testigo & $1,09 \mathrm{~b}$ & $1,15 \mathrm{~b}$ \\
\hline (1) Letras distintas en la misma columna indican diferencias estadísticamente \\
significativas entre tratamientos (P $\leq 0,01)$.
\end{tabular}

bas profundidades presentaron valores inferiores (Cuadro 3).

Los resultados de COT y de las distintas fracciones ( $\mathrm{COH}$ y $\mathrm{COL})$, para cada tratamiento, se presentan en los Cuadros 4 y 5. Los valores de COT y COL hallados en $\mathrm{T}$ (en ambas profundidades de muestreo) mostraron diferencias estadísticamente significativas $(\mathrm{P} \leq 0,01)$ con respecto a SD y LV.

La Figura 1 representa la relación entre el carbono total y la densidad máxima en todos los tratamientos. Los resultados indican que la máxima compactabilidad está inversamente relacionada con el contenido de carbono orgánico del suelo; esto es coincidente con lo encontrado por Pecorari et al. (1993) y Thomas et al. (1996).

El análisis de los datos de PE mostró diferencias estadísticamente significativas entre tratamientos y profundidad, al igual que la interacción tratamiento $\mathrm{x}$ profundidad $(\mathrm{P} \leq 0,01)$. Para las observaciones en superficie, LV tuvo los valores más elevados $(23,01 \%)$, siguiendo T con $16,06 \%$ y SD con $8,77 \%$. En la profundidad de $6-20 \mathrm{~cm}$, los valores más elevados correspondieron a $\mathrm{T}(11,71 \%)$, y los menores a SD $(4,09 \%)$, correspondiendo a LV un valor intermedio $(9,17 \%)$.

En la Figura 2, se muestra la relación entre CR y porosidad estructural (PE). De acuerdo con el análisis de regresión $\left(\mathrm{r}^{2}=0,869\right)$, a mayor PE del suelo corresponde una menor $\mathrm{CR}$.

Cuadro 2. Densidad máxima $\left(\mathrm{Mg} \mathrm{m}^{-3}\right)$ y contenido de agua (\%) crítico del suelo en superficie y profundidad, en los tratamientos siembra directa, labranza vertical y testigo ${ }^{(1)}$.

\begin{tabular}{|c|c|c|}
\hline Tratamiento & Densidad & Contenido de agua \\
\hline & \multicolumn{2}{|c|}{ Superficie $(0-6 \mathrm{~cm})$} \\
\hline Siembra directa & $1,488 \mathrm{a}$ & $18,98 \mathrm{a}$ \\
\hline Labranza vertical & $1,506 \mathrm{a}$ & $18,12 \mathrm{a}$ \\
\hline \multirow[t]{2}{*}{ Testigo } & $1,408 \mathrm{~b}$ & $18,80 \mathrm{a}$ \\
\hline & \multicolumn{2}{|c|}{ Profundidad $(6-20 \mathrm{~cm})$} \\
\hline Siembra directa & $1,572 \mathrm{a}$ & $21,07 \mathrm{a}$ \\
\hline Labranza vertical & $1,542 \mathrm{a}$ & $23,12 b$ \\
\hline Testigo & $1,495 b$ & $23,55 b$ \\
\hline
\end{tabular}


La ecuación de regresión obtenida se detalla a continuación:

$\operatorname{PE}(\%)=85,30314-0,91673 \times \mathrm{CR}\left(\mathrm{r}^{2}=0,869\right)$.

La presencia de residuos orgánicos posee un efecto protector frente a la compactación a campo. Dentro de la materia orgánica, la fracción ligada adquiere una mayor importancia debido a que aumenta la porosidad textural, modificando el comportamiento global de los suelos frente a la compactación (Pecorari et al., 1993).

Cuadro 3. Compactación relativa del suelo (\%) a ambas profundidades de muestreo, en los tratamientos siembra directa, labranza vertical y testigo ${ }^{(1)}$.

\begin{tabular}{lcc}
\hline Tratamiento & \multicolumn{2}{c}{ Profundidad $(\mathrm{cm})$} \\
\cline { 2 - 3 } & $0-6$ & $10-16$ \\
\hline Siembra directa & $82,00 \mathrm{a}$ & $87,15 \mathrm{a}$ \\
Labranza vertical & $69,06 \mathrm{~b}$ & $85,60 \mathrm{a}$ \\
Testigo & $77,41 \mathrm{~b}$ & $76,92 \mathrm{~b}$ \\
\hline (1) Letras distintas en la misma columna indican diferencias estadísticamente \\
significativas entre tratamientos (P $\mathbf{P} 0,01)$.
\end{tabular}

Cuadro 4. Carbono orgánico total (COT), carbono orgánico humificado $(\mathrm{COH})$ y carbono orgánico libre (COL) en superficie (0-6 cm), en los tratamientos siembra directa, labranza vertical y testigo ${ }^{(1)}$.

\begin{tabular}{|c|c|c|c|}
\hline Tratamiento & COT & $\mathrm{COH}$ & $\mathrm{COL}$ \\
\hline & \multicolumn{3}{|c|}{--------' } \\
\hline Siembra directa & $2,05 \mathrm{~b}$ & $1,53 b$ & $0,52 b$ \\
\hline Labranza vertical & $1,75 b$ & $1,34 \mathrm{~b}$ & $0,41 \mathrm{~b}$ \\
\hline Testigo & $4,03 \mathrm{a}$ & $2,43 a$ & $1,60 \mathrm{a}$ \\
\hline
\end{tabular}

Cuadro 5. Carbono orgánico total (COT), carbono orgánico humificado $(\mathrm{COH})$ y carbono orgánico libre (COL) en profundidad $(6-20 \mathrm{~cm})$, en los tratamientos siembra directa, labranza vertical y testigo ${ }^{(1)}$.

\begin{tabular}{lccc}
\hline Tratamiento & COT & COH & COL \\
\hline & - & -9 & -------- \\
Siembra directa & $1,48 \mathrm{~b}$ & $0,80 \mathrm{~b}$ & $0,68 \mathrm{~b}$ \\
Labranza vertical & $1,58 \mathrm{~b}$ & $1,10 \mathrm{a}$ & $0,48 \mathrm{~b}$ \\
Testigo & $2,29 \mathrm{a}$ & $1,26 \mathrm{a}$ & $1,03 \mathrm{a}$ \\
\hline (1) Letras distintas para una misma columna indican & diferencias \\
estadisticamente significativas entre tratamientos (P $\leq 0,01)$.
\end{tabular}

Según diversos autores, cuando la compactación relativa supera el $86-90 \%$ (dependiendo de la textura del suelo), se produce una reducción importante de la proporción de macroporos, afectando al normal movimiento de los fluidos. Esto estaría relacionado a un menor crecimiento y rendimiento de los cultivos (Carter, 1990; Lipiec et al., 1991). En nuestro ensayo, si bien SD y LV presentaron valores de

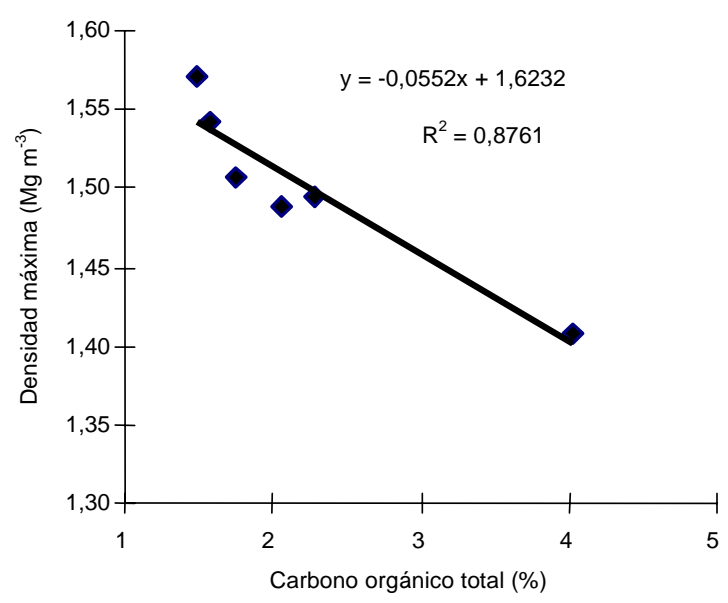

Figura 1. Relación entre el contenido de carbono orgánico total y la densidad del suelo máxima obtenida por el test Proctor, para los tratamientos siembra directa, labranza vertical y testigo.

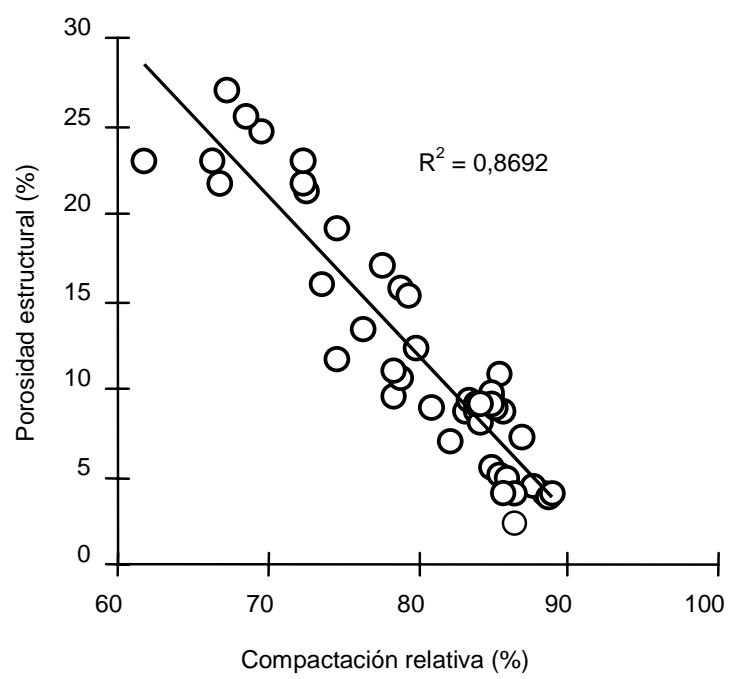

Figura 2. Relación entre porosidad estructural y compactación relativa en ambas profundidades, en los tratamientos siembra directa, labranza vertical y testigo. 
CR elevados a los 10-16 $\mathrm{cm}$ de profundidad $(87,15 \%$ y $85,6 \%$ para SD y LV, respectivamente), el crecimiento y rendimiento del cultivo bajo SD se vio notoriamente afectado (fue el $47,88 \%$ del obtenido en LV). Posiblemente, el hecho de encontrarse en SD una capa de suelo en superficie compactada $(\mathrm{CR}=82 \%)$, a diferencia de la CR hallada en LV $(69,06 \%)$, pudo ser un factor que influyó sobre el crecimiento de las raíces, determinando la reducción de los rendimientos.

Voorhees \& Lindstrom (1984) sostienen que los suelos bajo SD tienen menor porosidad total durante aproximadamente los primeros cinco años de iniciado el sistema y luego comienza a incrementarse hasta llegar a un estado de equilibrio. Los agregados de suelos bajo $\mathrm{SD}$, con el tiempo serían más porosos dado que contienen mayor proporción de restos orgánicos y canales como resultado de la actividad biológica.

En la Figura 3 se observan los mapas con los estados estructurales obtenidos en cada situación. En el tratamiento SD fue necesario separar por sectores y realizar cartas estructurales diferentes debido a que el cultivo estaba muy desparejo, presentando zonas
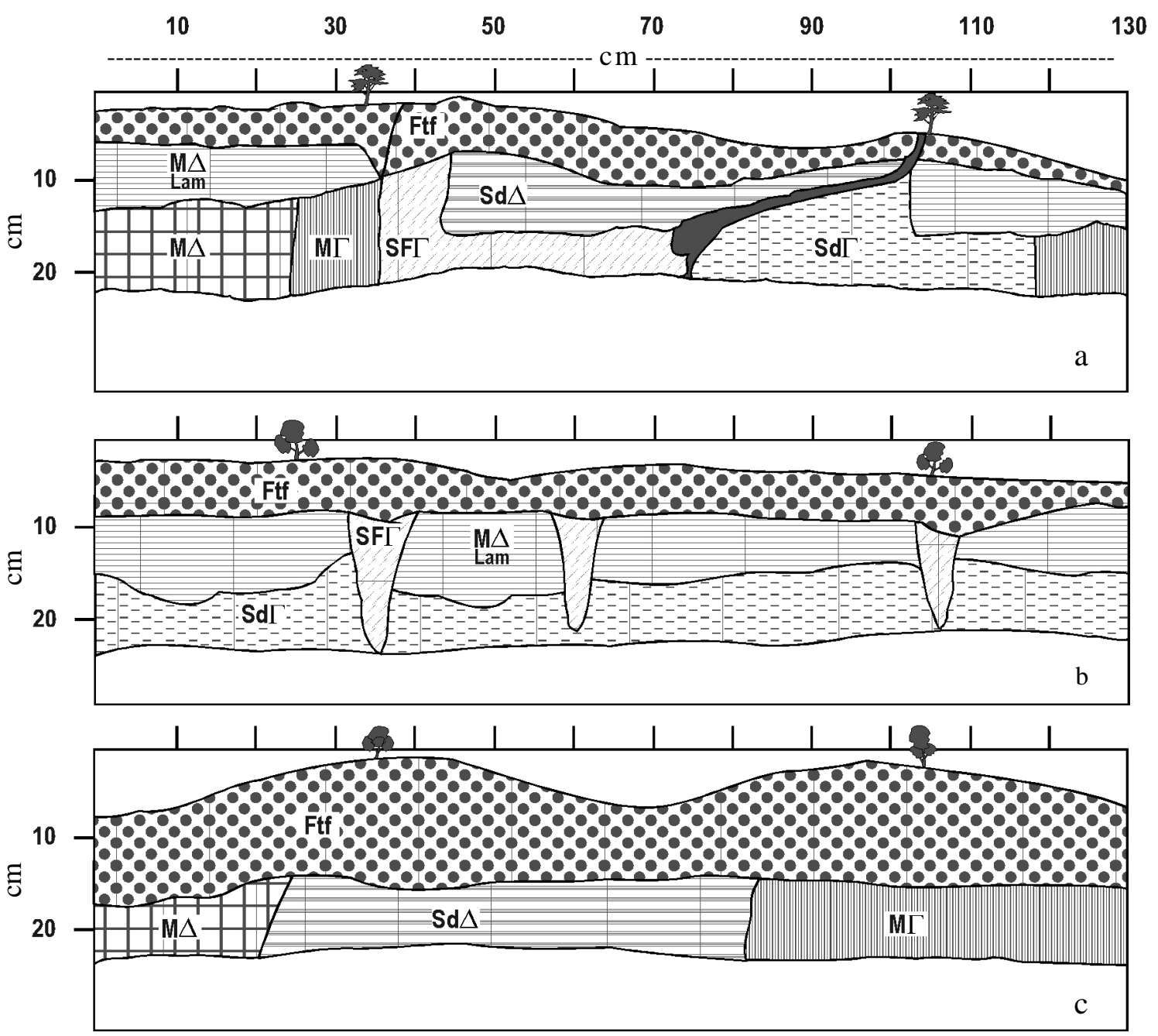

Figura 3. Perfil cultural en sector de siembra directa con predominio de plantas de menor desarrollo (a), de mejor desarrollo (b) y perfil cultural en labranza vertical (c). 
con plantas cloróticas y de menor altura. En el perfil correspondiente a SD con predominio de plantas de menor desarrollo (altura 0,7-0,9 m) se observaron sectores masivos $(\mathrm{M})$ y terrones soldados $(\mathrm{Sd}) \sin$ porosidad interna, con caras de ruptura lisas y de cohesión elevada $(\Delta)$ en un $41,4 \%$ (Figura $3 a$ ).

En el sector de SD con plantas de mejor desarrollo (altura promedio $1 \mathrm{~m}$ ) y mayor proporción de nódulos, se determinó un $32,3 \%$ de estado masivo de muy baja porosidad con estructura laminar ( $\mathrm{M} \Delta$ lam.), siendo una capa menos compactada que $\mathrm{M} \Delta$ (Figura $3 \mathrm{~b}$ ). Se tomaron muestras con cilindro para determinar la densidad de volumen en la zona M $\Delta$ lam., siendo los valores promedio obtenidos $1,37 \mathrm{Mg} \mathrm{m}^{-3}$. En ambos casos se observó la presencia en superficie de una capa de 2-4 cm de espesor de elementos finos y restos de materia orgánica en diferentes estados de descomposición.

De Battista et al. (1992), trabajando sobre suelos de Pergamino con diferentes labranzas, hallaron un $28,5 \%$ de estado interno $\Delta$ en lotes bajo SD provenientes de agricultura. La mayor proporción de estado $\Delta$ en SD pone de manifiesto que este sistema de labranza no mejoraría las condiciones físicas del suelo, sino que mantiene una condición de equilibrio; al menos en el mediano y corto plazo. El estado estructural del suelo al momento de iniciar el sistema de labranza juega un rol fundamental en la evolución de las propiedades físicas.

El perfil realizado en LV contrasta con los otros dos, ya que el 58,8\% del horizonte Ap presenta un estado fragmentario con predominio de tierra fina (Ftf) (Figura 3c). En esta capa se observó un incremento en la proporción de raíces y mayor cantidad de nódulos que en SD. Por debajo de la capa Ftf se observó una zona más compactada con un $27,8 \%$ de sectores $\mathrm{M}$ y $\mathrm{Sd}$ con estado interno $\Delta$, zona que coincide con el fondo de trabajo que podría convertirse en un impedimento para la penetración de las raíces y para la circulación de agua, aire y nutrimentos. El desarrollo de las plantas (altura promedio $1,2 \mathrm{~m}$ ) y el contenido de agua al momento de floración - fructificación fue mayor en LV.

En el Cuadro 6 se detallan los diferentes estados estructurales según la disposición de los terrones (2o nivel) y según el reagrupamiento de las modalidades de disposición (3o nivel) para los tratamientos SD y LV.
La elevada proporción de estado compacto en SD (63,9\% y $62 \%$ en ambos perfiles realizados) indica una mayor compactación con la consecuente deficiencia en la exploración de raíces. Estas zonas sin discontinuidades estructurales pueden tener una incidencia negativa sobre el desarrollo del cultivo, fundamentalmente en los suelos con baja fertilidad química y deficiencias hídricas.

Por el contrario, LV presentó un 58,8\% de estado óptimo, condición que favoreció el desarrollo de raíces en superficie, aunque como se mencionó anteriormente por debajo de esa capa se detectó una zona compactada.

El contenido de agua del suelo al inicio y al momento de floración-fructificación del cultivo se observa en el Cuadro 7. Hubo diferencias estadísticamente significativas $(\mathrm{P} \leq 0,01)$ entre tratamientos de labranza y entre profundidades, para ambas épocas de muestreo. Un mayor contenido de agua al inicio del cultivo en SD, puede estar relacionado a las menores pérdidas de agua por evaporación debido a la presencia de residuos vegetales en superficie y a la falta de laboreo del suelo (Zhai et al., 1990; Chagas et al., 1994). En la etapa de floración-fructificación el tratamiento LV presentó mayor HG que SD.

Cuadro 6. Estados estructurales (\%) de los tratamientos siembra directa y labranza vertical.

\begin{tabular}{|c|c|c|c|}
\hline Estados estructurales $^{(1)}$ & $\begin{array}{l}\text { Siembra } \\
\operatorname{directa}^{(2)}\end{array}$ & $\begin{array}{l}\text { Siembra } \\
\text { directa }^{(3)}\end{array}$ & $\begin{array}{c}\text { Labranza } \\
\text { vertical }\end{array}$ \\
\hline $\mathrm{Ftf}$ & 20,6 & 25,2 & 58,8 \\
\hline $\mathrm{SF \Gamma}$ & 15,5 & 12,8 & \\
\hline Total & 36,1 & 38,00 & 58,8 \\
\hline $\mathrm{M} \Delta$ & 10,3 & & 6,2 \\
\hline $\mathrm{M} \Delta$ laminar & 18,4 & 32,3 & \\
\hline МГ & 8,1 & 29,7 & 13,4 \\
\hline $\mathrm{Sd} \Delta$ & 12,7 & & 21,6 \\
\hline $\mathrm{Sd} \Gamma$ & 14,4 & & \\
\hline Total & 63,9 & 62,00 & 41,2 \\
\hline \multicolumn{4}{|c|}{$\begin{array}{l}\text { (1) Segundo nível: disposición de los terrones (Ftf: estado fragmentario con } \\
\text { predominio de tierra fina; SF: estado fragmentario con terrones individu- } \\
\text { alizados; M: estado masivo; Sd: terrones dificilmente discernibles); tercer } \\
\text { nível: reagrupamiento de las modalidades de disposición en óptimo } \\
\text { (Ftf, SF) y compacto (M, Sd). }{ }^{(2)} \text { Plantas cloróticas y menor altura. }{ }^{(3)} \text { Plan- } \\
\text { tas de mayor desarrollo. }\end{array}$} \\
\hline
\end{tabular}


El crecimiento y la cobertura por parte del cultivo en SD fue muy baja, con lo cual las pérdidas por evaporación fueron mayores que en LV. Además, como consecuencia de una mayor compactación en superficie, pudo haber ingresado menor cantidad de agua que en LV.

Los datos de rendimiento en grano fueron significativamente mayores en LV (3.550 kg ha-1) con respecto a lo obtenido en $\mathrm{SD}\left(1.700 \mathrm{~kg} \mathrm{ha}^{-1}\right)$ $(\mathrm{P} \leq 0,01)$. Estas diferencias podrían deberse a un menor desarrollo de las raíces en SD como consecuencia de algún impedimento mecánico que afectó al abastecimiento de agua y nutrimentos, resultados que coinciden con los obtenidos por Cornish \& Lymbery (1987) y Schmidt \& Belford (1994). Estos autores que trabajaron sobre suelos susceptibles a la compactación hallaron una correlación positiva entre rendimiento del cultivo y profundidad de remoción del suelo en la línea de siembra; proponiendo romper las capas densas por medio de alguna labor para así favorecer el crecimiento de raíces antes de implantar cultivos bajo SD.

El ensayo se encuentra en el sexto año de iniciado, período que puede considerarse suficiente como para que ocurra una estabilización de las propiedades físicas del suelo. La situación evaluada en T representaría la condición inicial del suelo, no degradada por la agricultura continua. Las condiciones físicas del suelo al momento de iniciar el sistema con SD son un factor determinante en la evolución de las mismas a lo largo del tiempo. La implementación de pasturas consorciadas, la incorporación de importantes volúmenes de rastrojos y la reducción de la compactación de la capa superficial serían prácticas

Cuadro 7. Humedad gravimétrica (\%) promedio medida al inicio del cultivo (10/12/96) y en la etapa de floración (5/3/97) a ambas profundidades de muestreo en los tratamientos siembra directa y labranza vertical ${ }^{(1)}$.

\begin{tabular}{llllll}
\hline Tratamiento & \multicolumn{2}{c}{ Inicio del cultivo } & & \multicolumn{2}{c}{ Floración } \\
\cline { 2 - 3 } \cline { 5 - 6 } & $0-10 \mathrm{~cm}$ & $10-20 \mathrm{~cm}$ & & $0-10 \mathrm{~cm}$ & $10-20 \mathrm{~cm}$ \\
\hline Siembra directa & $22,68 \mathrm{a}$ & $23,13 \mathrm{a}$ & $23,45 \mathrm{~b}$ & $22,11 \mathrm{~b}$ \\
Labranza vertical & $16,10 \mathrm{~b}$ & $17,53 \mathrm{~b}$ & & $30,20 \mathrm{a}$ & $27,70 \mathrm{a}$ \\
\hline $\begin{array}{llll}\text { (1) Letras distintas en la misma columna indican diferencias estadísticamente } \\
\text { significativas entre tratamientos }(\mathrm{P} \leq 0,01) .\end{array}$
\end{tabular}

de manejo a tener en cuenta ya que el éxito de la implementación de la SD depende del estado físico inicial del suelo utilizado.

\section{Conclusiones}

1. SD presenta en superficie menor porosidad total y estructural que LV, factores que pueden incidir sobre el volumen de exploración de raíces y el movimiento de agua y aire.

2. Los resultados de CR y los mapas estructurales denotan mayor compactación en $\mathrm{SD}$, mientras que en LV se observa en superficie predominio de tierra fina.

3. Los resultados de porosidad total, porosidad estructural y carbono orgánico hallados en $\mathrm{T}$ son significativamente superiores en comparación con SD y LV.

4. El rendimiento del cultivo de soja bajo SD es menor del obtenido en LV, probablemente como resultado de la mayor compactación observada en este tratamiento.

\section{Agradecimientos}

A los Sres. Facundo Baldassin y Hernan Gassmann (Auxiliares de docencia de Edafología, Facultad de Ciencias Agrarias, Universidad Nacional de Rosario) por la participación en las tareas realizadas a campo y las determinaciones de laboratorio.

\section{Referencias}

ANDRIULO, A.; GALANTTINI, J.; PECORARI, C.; TORIONI, E. Materia orgánica del suelo en la región pampeana argentina. I. Un método de fraccionamiento por tamizado. Agrochimica, Pisa, v. 34, n. 5/6, p. 475-489, 1990.

BLACK, C. A. Organic carbon. In: BLACK, C. A. (Ed.). Methods of soil analysis. Madison : American Society of Agronomy, 1965. pt. 2. (ASA. Agronomy, 9).

BLAKE, G. R.; HARTGE, K. H. Bulk density. In: KLUTE, A. (Ed.). Methods of soil analysis. 2. ed. Madison : American Society of Agronomy, 1986. pt. 1, p. 363-375. (ASA. Agronomy, 9).

CARTER, M. R. Relative measures of soil bulk density to characterize compaction in tillage studies on fine sandy 
loams. Canadian Journal of Soil Science, Ottawa, v. 70 p. 425-433, 1990.

CHAGAS, C. I.; MARELLI, H. J.; SANTANATOGLIA, O. J. Propiedades físicas y contenido hídrico de un Argiudol típico bajo tres sistemas de labranza. Ciencia del Suelo, Buenos Aires, v. 12, p. 11-16, 1994.

CORNISH, P. S.; LYMBERY, J. R. Reduced early growth of direct drilled wheat in southern New South Wales: causes and consequences. Australian Journal of Experimental Agriculture, Collingwood, v. 27, p. 869-880, 1987.

COSCIA, A. A. La agriculturización en la región pampeana. In: DEGRADACIÓN de suelos por intensificación de la agricultura. Rafaela : Instituto Nacional de Tecnología Agropecuaria, 1989. 179 p. (Publicación Miscelánea, 47).

DE BATTISTA, J. J.; ANDRIULO, A.; PECORARI, C. El perfil cultural: un método para la evaluación de sistemas de cultivos. Ciencia del Suelo, Buenos Aires, v. 10/ 11, p. 89-93, 1992.

GARDNER, W. H. Water content. In: KLUTE, A. (Ed.). Methods of soil analysis. 2. ed. Madison : American Society of Agronomy, 1986. pt. 1, p. 493-544. (ASA. Agronomy, 9).

LAL, R.; MAHBOUBI, A. A.; FAUSEY, N. R. Long-term tillage and rotation effects on properties of a Central Ohio soil. Soil Science Society of America Journal, Madison, v. 58, p. 517-522, 1994

LARNEY, F. J.; KLADIVKO, E. J. Soil strength properties under four tillage systems at three long-term study sites in Indiana. Soil Science Society of America Journal, Madison, v. 53, p. 1539-1545, 1989.

LIPIEC, J.; HAKANSSON, I.; TARKIEWICZ, S.; KASSOWSKI, J. Soil physical properties and growth of spring barley as related to the degree of compactness of two soils. Soil and Tillage Research, Amsterdam, v. 19, p. 307-317, 1991.

MAHBOUBI, A. A.; LAL, R.; FAUSSEY, N. R. Twenty-eight years of tillage effects on two soils in Ohio. Soil Science Society of America Journal, Madison, v. 57, p. 506-512, 1993.

MANICHON, H. Influence des systémes de culture sur le profil cultural: élaboration d'une méthode de diagnostic basée sur 1'observation morphologique. Paris : Institut National Agronomique, 1982. 311 p. Thèse de Doctorat.
MANICHON, H. Observation morphologique de l'état structural et mise en évidence d'effects de compactage des horizons travaillés. In: MONNIER, G.; GOSS, M. J. (Ed.). Soil compaction and regeneration. Rotterdam : Balkema, 1987. p. 39-52.

MARELLI, H. La siembra directa en la Argentina. In: INSTITUTO NACIONAL DE TECNOLOGÍA AGROPECUARIA. Estación Experimental Agropecuaria (Marco Juárez, Argentina). Proyecto de investigación estratégica: cultivos sin labranza. Marcos Juárez, 1994. 11 p. (Hoja Informativa, 273).

MONNIER, G.; STENGEL, P.; FIES, J. C. Une méthode de mesure de la densité apparente de petits agglomérats terreux: application a l'analyse de porosité du sol. Annales Agronomiques, Versailles, v. 24, p. 533-545, 1973.

NESMITH, D. S.; RADCLIFFE, D. E.; HARGROVE, W. L.; CLARK, R. L.; TOLLNER, E. W. Soil compaction in double-cropped wheat and soybeans on an Ultisol. Soil Science Society of America Journal, Madison, v. 51, p. 183-186, 1987.

NETER, J.; WASSERMAN, W.; KUTNER, M. H. Applied linear statistical models: regression, analysis of variance and experimental designs. 2. ed. Homewood : R. D. Irwin, $1985.1127 \mathrm{p}$

OUSSIBLE, M.; CROOKSTON, R. K.; LARSON, W. E. Subsurface compaction reduces the root and shoot growth and grain yield of wheat. Agronomy Journal, Madison, v. 84, p. $34-38,1992$.

PECORARI, C. Inestabilidad estructural de los suelos de la Pampa Ondulada. Pergamino : Instituto Nacional de Tecnología Agropecuaria, 1988. 16 p. (Informe Técnico, 216).

PECORARI, C.; ANDRIULO, A.; AUSILIO, A. Aptitud físico-mecánica de los suelos para el laboreo. II: Efectos de la textura y de la materia orgánica sobre la densidad aparente máxima de algunos suelos de la región pampeana argentina. Pergamino : Instituto Nacional de Tecnología Agropecuaria, 1993. 16 p. (Informe Técnico, 281).

PIZARRO, J. B.; TORT, M. I.; BEARZOTTI, S.; CACCIAMANI, M. A. Nuevas estrategias de producción y su relación con el recurso suelo. Pergamino : Instituto Nacional de Tecnología Agropecuaria, 1992. 20 p. (Informe Técnico, 268).

PROCTOR, R. R. Fundamental principles of soil compaction. Engineering News-Record, New York, v. 11, n. 9 , p. $148-156,1933$. 
SAS INSTITUTE (Cary, Estados Unidos). SAS user's guide: Statistics version. 5. ed. Cary, 1985. t. 2.

SCHMIDT, C. P.; BELFORD, R. K. Increasing the depth of soil disturbance increases yields of direct drilled wheat on the sandplain soil of Western Australia. Australian Journal of Experimental Agriculture, Collingwood, v. 34, p. 777-781, 1994.

SOANE, B. D.; BLACKWELL, P. S.; DICKSON, J. W.; PAINTER, D. J. Compaction by agricultural vehicles: a review. 1. Soil and wheel characteristics. Soil and Tillage Research, Amsterdam, v. 1, p. 207-237, 1981.

STEEL, R.; TORRIE, J. Bioestadística: principios y procedimientos. México : McGraw-Hill, 1986. 622 p.
THOMAS, G. W.; HASZLER, G. R.; BLEVINS, R. L. The effects of organic matter and tillage on maximum compactability of soils using the Proctor test. Soil Science, Baltimore, v. 161, p. 502-508, 1996.

VOORHEES, W. B.; LINDSTROM, M. J. Long-term effects of tillage method on soil tilt independent of wheel traffic compaction. Soil Science Society of America Journal, Madison, v. 48, p. 152-156, 1984.

ZHAI, R.; KACHANOSKI, R. G.; VORONEY, R. P. Tillage effects on the spatial and temporal variation of soil water. Soil Science Society of America Journal, Madison, v. 54, p. 186-192, 1990. 